\title{
Using neural network for drowsiness detection based on EEG signals and optimization in the selection of its features using genetic algorithm
}

Utilización de la red neuronal para la detección de somnolencia basada en señales de EEG y optimización en la selección de sus características mediante algoritmo genético
Author:

Sepehr Sarabi ${ }^{1}$

Milad Asadnejad ${ }^{2 *}$

Saman Rajabi ${ }^{3}$

\section{SCIENTIFIC RESEARCH}

How to cite this paper:

Sarabi S., Asadnejad M. and Rajab S., Using neural network for drowsiness detection based on EEG signals and optimization in the selection of its features using genetic algorithm, Iran. Innovaciencia 2020; 8 (1): 1-9. DOI: http://dx.doi.org/ 10.15649/2346075X.1004

Reception date:

Received: 01 May 2020

Accepted: 01 August 2020

Published: 01 December 2020

\section{ABSTRACT}

Introduction: One of the major causes of traffic accidents is driver's drowsiness. For this reason, detecting whether the driver's eyes are open or closed is one of the critical factors in reducing road deaths. Materials and Methods: One way to detect whether your eyes are open or closed is to use EEG signals. EEG signals are obtained from the recording of electrical activity in the human brain. The present study uses a neural network that is applied to the driver's EEG signals to detect whether the eye is open or closed. The data of the EEG signals used in this paper consist of 14 features that are based on a statistical population of 600 people. Results and Discussion: Various neural network algorithms have been implemented for clustering these data into two classes of open or closed eyes, which are described in this paper. Perceptron neural network and radial base neural network (RBF) are two types of networks used in this paper. Also, in order to improve the execution speed and reduce the occupied space of the microcontroller, the genetic algorithm method has been used to optimize the fitting function of Fisher's discriminant rate, in which the optimized function provides better results in the less occupied time and space.

Keywords:

Drowsiness, EEG Signal, Optimization, Genetic Algorithm, Neural Network, Perceptron, Radial Base Functions

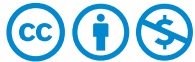

CC BY-NC 4.0
1 Department of Medical Engineering, Faculty of Engineering, Tabriz Branch, Islamic Azad University, Tabriz, Iran

2* Department of Medical Engineering, Faculty of Engineering, Tabriz Branch, Islamic Azad University, Tabriz, Iran Corresponding author: Milad Asadnejad Email: stu.m.asadnejad@iaut.ac.ir

${ }^{3}$ Department of Medical Engineering, Faculty of Engineering, Seraj Higher Education Institute, Tabriz, Iran 


\section{INTRODUCTION}

According to the World Health Organization, traffic deaths in the world have reached 1.24 million people a year, which is expected to increase to 3.6 million by 2030 . According to global statistics, most road accidents are related to the drowsiness of drivers. Studies show that more than $20 \%$ of road casualties are caused by driver drowsiness. Therefore, designing a system for drowsiness detection is one of the best ways to reduce statistics considerably. Among the methods that can be used to detect drowsiness is the processing of vital signals or the processing of driver images. One of the disadvantages of processing driver images is driver's distraction and the unwillingness to permanently record the driver's image. EEG signals are one of the vital signals that can be used to detect whether the eye is open or closed.

Electroencephalography (EEG) is the recording of the brain's electrical activity by placing surface electrodes on the scalp using a non-invasive method. In general, in an EEG system, the electrical effect of the activity of the brain's neurons is transmitted to the device through electrodes placed on the scalp, and after amplifying and removing the noise, it is recorded and displayed as a time signal. The recorded signal can be analyzed directly or after computer processing by an expert. The EEG signals range between 3 and $70 \mathrm{~Hz}$, and the recording device usually includes 8, 16, and 32 channels, which are placed on the patient's scalp by special leads. $[1,2]$

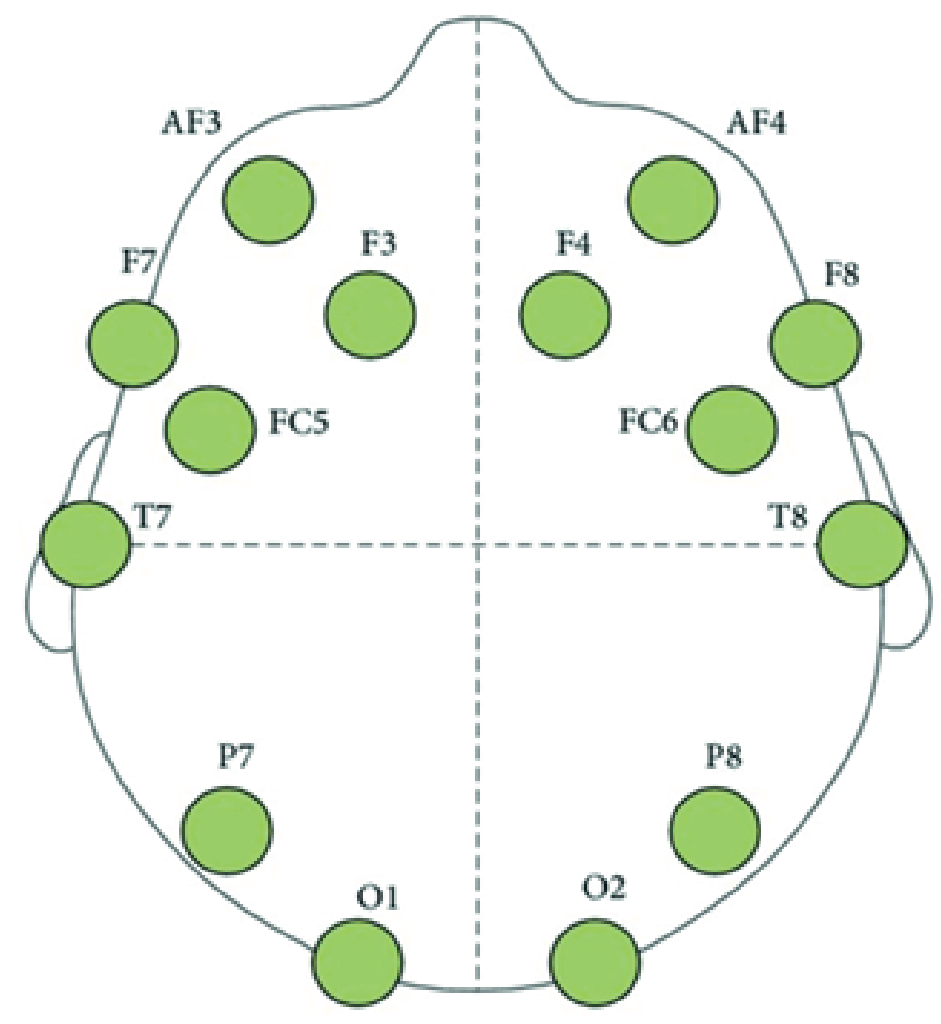

Figure 1: Location of EEG leads on the scalp [3]

Interpretation of Electroencephalography is performed by a neurologist. These results are based on the amplitude of the wave fluctuations and finding defect patterns in the Electroencephalography. If the Electroencephalography is normal, these waves will have a regular pattern without damage. However, in cases where the patient moves or open his eye or making another movement, the Electroencephalography pattern changes. 


\section{METHODS}

To detect drowsiness in driving, EEG signals should be detected using artificial intelligence methods that have high speed and accuracy. Among the methods used in this study to classify the open and closed state of the eye are the perceptron neural network and the radial base function (RBF).

The data collected in this paper is measured using a continuous EEG meter by EEG neuroheadset Emotive device for 117 seconds. The statistical population is 600 people, with 14 features of brain channels received by leads, and two states of open or closed eyes are used as a data class.

\subsection{Artificial neural network}

The artificial neural network is a simulation of the human brain that solves many problems without the need to know how to analyze them and by finding a suitable pattern or mathematical model. Correct training is the only requirement for artificial neural networks to function properly, which is similar to the function of the neural networks of the human brain, doing a lot of work without being aware of its relationship. Using the neural network algorithm, different and complex models can be identified. For example, we can do well-structured classifications or clustering large data. Different types of neural networks include the perceptron neural network or the radial base function neural network (RBF), each of which has some advantages or disadvantages. [4, 5]

The present study refers to the eyes opening or closing using a variety of neural networks and optimization of the results, speed and reducing the number of features.

\subsubsection{Perceptron Neural Network}

A perceptron neural network consists of three layers of input, output, and hidden, each layer containing a group of nerve cells that are generally associated with all the neurons in the other layer. One of the most important applications of neural networks is classification of statistical data. Several neural networks have been introduced in classification. The relationship between the perceptron algorithm is as follows. $[6,7]$

$$
w \cdot x=\sum_{i=1}^{m} w_{i} \cdot x_{i}+b
$$

Where $\mathrm{w}$ is the impact value of each of the features called weight and $\mathrm{b}$ is the bias and $\mathrm{x}$ is the input vector.

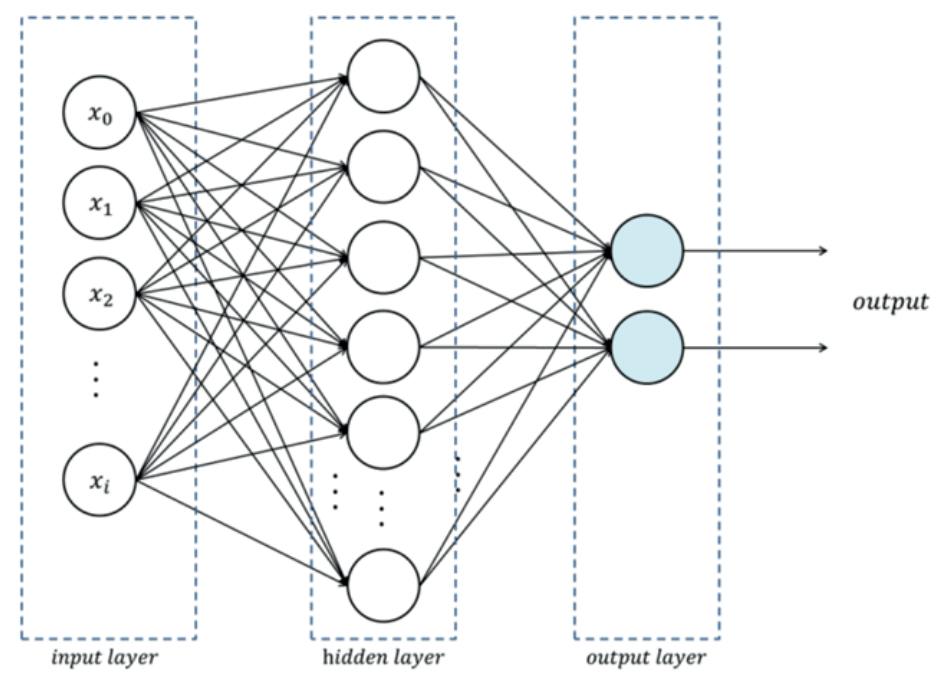

Figure 2: Perceptron neural network [8] 
In perceptron neural network, by changing the number of layers, the number of neurons, and the type of transform function, the obtained results are different. In this paper, this type of network has been used to detect whether the eye is open or closed, in which by changing the number of neurons and the type of transform function, the obtained results and its execution time will be different.

Tansig and Losing functions are different types of transform functions.

$$
\begin{aligned}
& \operatorname{Tan} \operatorname{Sig}(x)=1 /\left(1+e^{(-2 x)}\right)-1 \\
& \operatorname{Logsig}(x)=1 /\left(1+e^{(-x)}\right)
\end{aligned}
$$

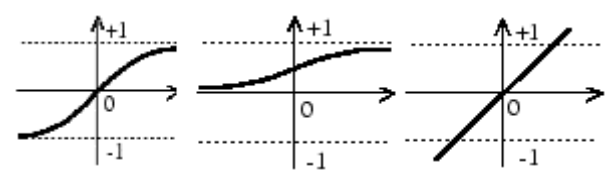

Figure 3: Some examples of conventional transform functions [9]

Different results from the Perceptron neural network with Tansig and Losing transform functions are as follows. The results of the Tansig transform function with the different number of neurons are shown in Table 1 and its block form is shown in Figure 4.

Table 1: The results of the perceptron neural network using Tansig transform function

\begin{tabular}{ccccc}
\hline Number of neurons & 10 & 20 & 30 & 40 \\
\hline$(\%)$ Accurate classification rate & 98.70 & 99.25 & 98.48 & 99.04 \\
\hline
\end{tabular}

According to CCR values in Table 1, the best solution is in the range of $30<$ number of neurons $<20$.

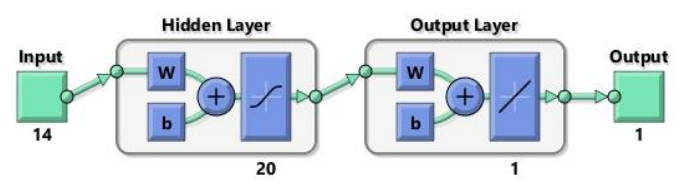

Figure 4: Neural network block with Tansig function

The results of the Logsig transform function with the number of different neurons are shown in Table 2 and its block form is shown in Figure 5.

Table 2. The results of the perceptron neural network in the Logsig transform function

\begin{tabular}{ccccc}
\hline Number of neurons & 10 & 20 & 30 & 40 \\
\hline$(\%)$ Accurate classification rate & 98.54 & 99.45 & 99.19 & 99.25 \\
\hline
\end{tabular}

According to the CCR values in Table 2, the best response is in the range of $30<$ number of neurons $<20$.

\section{Innovaciencia}




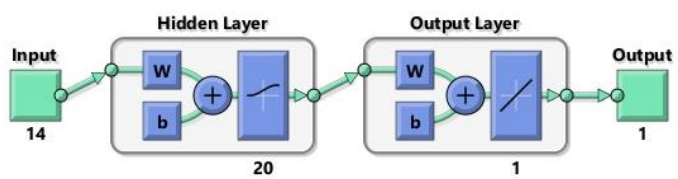

Figure 5. Neural network block with Logsig function

\subsubsection{Radial base function neural network}

The RBF neural network consists of neural cells that follow radial base functions. The network uses Gaussian functions to separate classes. In other words, the output of this system is a linear combination of radial base functions and input vectors. Variance and mean Gaussian functions are ordered to model the separator line. By changing the number of radial functions, the obtained results and the execution time of the plan are different $[10,11]$.

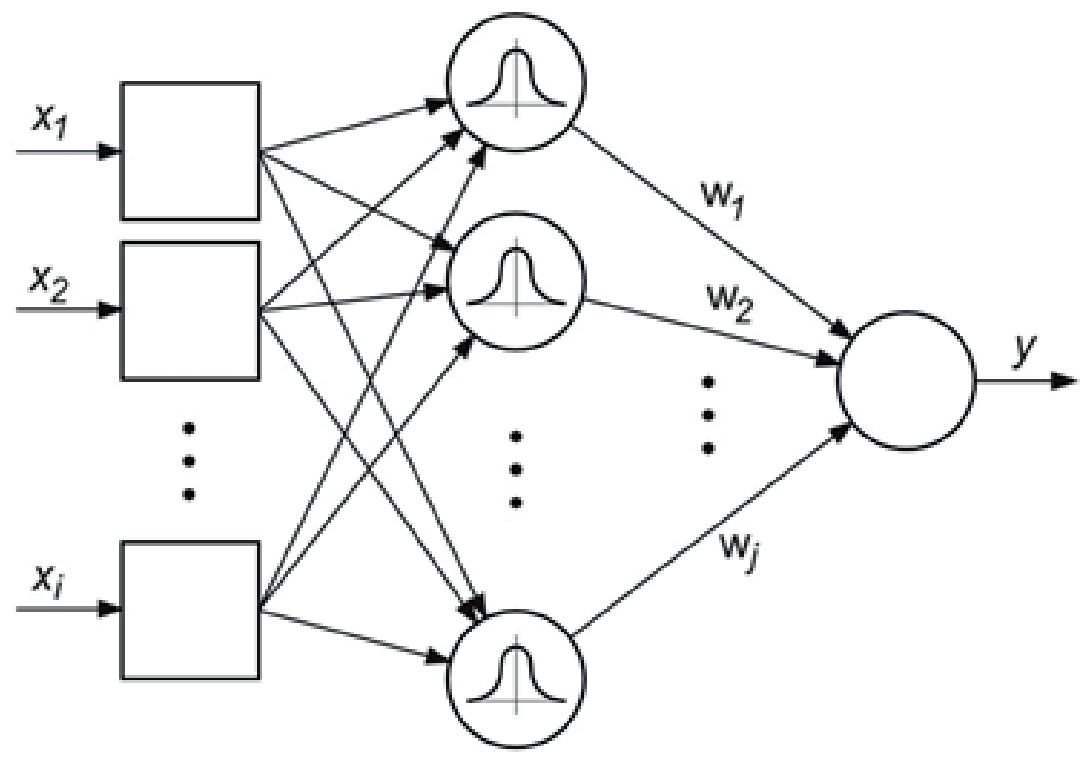

Figure 6: RBF neural network [12]

In RBF neural network, by changing the number of radial base functions, the obtained clustering rate is changed and the increase of the number of these functions is effective on its computation time.

Table 3. Results obtained from changing the number of radial functions

\begin{tabular}{ccccc}
\hline The number of radial base functions & 1 & 5 & 10 & 15 \\
\hline$(\%)$ Classification accurate rate & 93.24 & 99.82 & 99.99 & 100 \\
\hline
\end{tabular}

According to the obtained CCR, the best result in the number of radial functions is 15 .

Also, by Fisher's discriminant ratio, we can obtain the importance of each of 14 features in determining the open and close state of the eyes.

\subsection{Fisher's Discriminant Ratio (FDR)}

Fisher's discriminant ratio is used to determine the discrimination ratio of the classes from each other and the effect of each of the features on the amount of this discrimination and also is used for the possibility of the features combination for better discrimination [13]. In Fisher's method, for better 
discrimination of two classes, the shared region between two classes in their probability density function should be as small as possible and based on the concept of Gaussian functions parameters, it is clear that the higher the mean difference of the classes and the smaller the variance of the classes, the shared region gets smaller and the classes will have better discrimination [14].

The Fisher's Equation for the data with two classes can be expressed as:

$$
\operatorname{FDR}=\frac{\left(\mu_{1}-\mu_{2}\right)^{2}}{\left(\sigma_{1}^{2}-\sigma_{2}^{2}\right)}
$$

Where $(\mu)$ is the mean indicator and $(\sigma)$ is the variance indicator. The accurate classification discrimination rate is improved by increasing the Fisher's discriminant ratio, or the classification of two classes gets simpler.

The second application of Fisher's discriminant ratio is as the different features of samples as a linear combination form a feature beside each other. The features coefficients in this linear combination will change as the Fisher's discriminant ratio is maximized. Thus, besides increasing the discrimination ratio of two classes to have better classification, the number of features is reduced and the processing speed of the program should be increased [15].

As Fisher's discriminant ratio is based on the variance mean, it can not be optimized, unless we can add some values to the features. To find and optimize the unknown coefficients, optimization algorithms are used. Before dealing with the second application of Fisher's discriminant ratio, we should investigate one of the optimization algorithms for minimization or maximization of a function. To do this, one of the simplest and accurate algorithms such as genetic

FitnessFunction $=\frac{1}{\operatorname{FDR}}=\frac{\left(\mathrm{a}^{\prime *} \sigma 1^{*} \mathrm{a}+\mathrm{a}^{\prime *} \sigma 2 * \mathrm{a}\right)}{\left(\mathrm{a}^{\prime *}(\mu 1-\mu 2)^{*}(\mu 1-\mu 2)^{*} \mathrm{a}\right)}$ algorithm is investigated.

\subsection{Genetic algorithm}

Genetic algorithm is based on genetics and the survival of the best individual or its natural selection.

In genetic algorithms, the genetic evolution of the live creatures is simulated. In other words, a population of creatures is formed as in nature and by imposing on this set, we achieve an optimal set or an optimal creature $[16,17]$.

In brief, genetic algorithm is used to estimate the variables of a function for its minimizing. As genetic algorithm has minimizing rule, FDR equation in genetic algorithm is written as inversed. As the linear combination is based on two features, so a general coefficient called the coefficient matrix a is considered. This factor is also multiplied directly by the features mean when averaging. This coefficient is also multiplied using power 2 in the features variance when the variance is obtained (Equation 5).

Using the genetic algorithm implemented in MATLAB software, the values of the unknown coefficient and the values of the fitness function are simulated in the following diagram. 


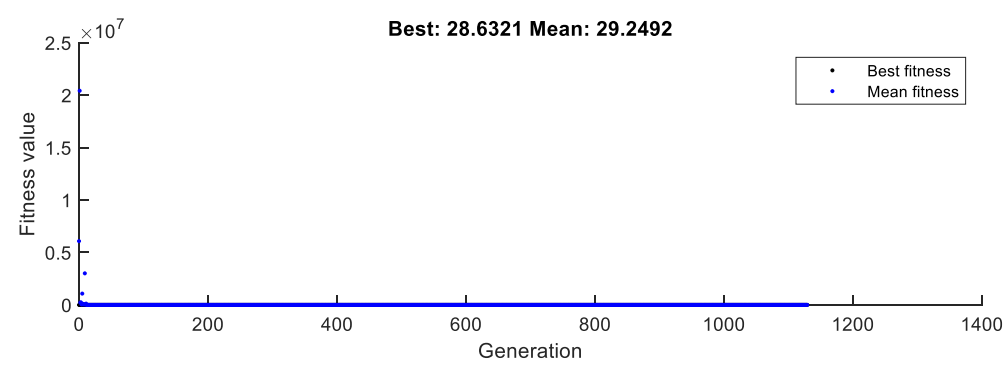

(a)

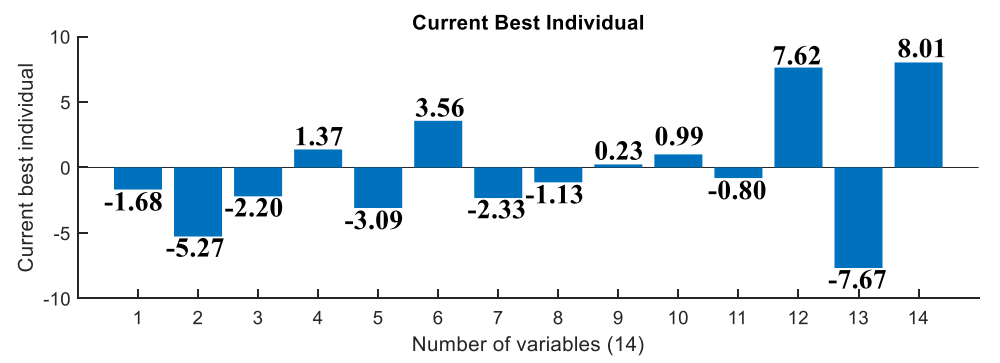

(b)

Figure 7: The diagram of the fitness function value (a) and the number of unknown coefficients (b)

Then, by multiplying the values of the coefficient a by the features matrix, the new data matrix with one feature is obtained that is placed in perceptron neural network for re-clustering.

The results with the number of different neurons are shown below

The results of the number of different neurons for perceptron neural network with the optimized matrix are shown in Table 4 and its block is depicted in Figure 8.

Table 4: The results of the perceptron neural network with optimized feature matrix

\begin{tabular}{ccccc}
\hline Number of neurons & 10 & 20 & 30 & 40 \\
\hline$(\%)$ Classification accurate ratio & 98.20 & 98.38 & 98.19 & 98.24 \\
\hline
\end{tabular}

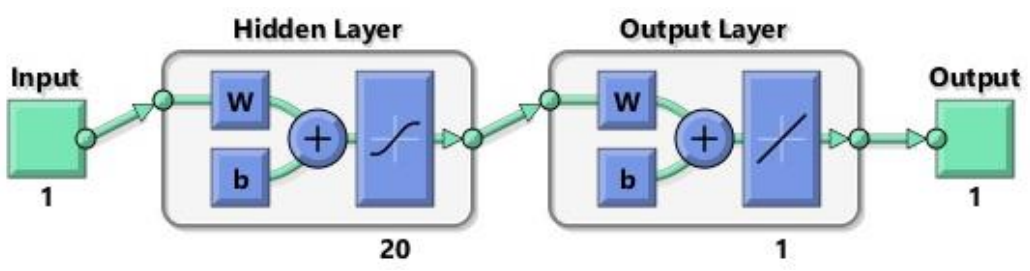

Figure 8: Neural network block with optimized matrix 


\section{CONCLUSION}

The open or closed clustering of the eye is implemented using EEG signal data by the perceptron neural network and the radial base functions neural network. In perceptron neural network, by changing the number of neurons and the type of transform function, the obtained results change and the best solution in the number of neurons is between 20 and 30 and the logsig transform function. In the radial base functions neural network, by the increase of the number of radial functions, the accurate clustering rate is also increased. Considering the implementation of neural networks on the microcontroller, the perceptron neural network can be easily run on the microcontroller via simulink environment. To reduce the execution time of the program and the space occupied in the microcontroller, the genetic algorithm method has been used to optimize the fitness function of the fisher's discrimination ratio, as by multiplying the coefficient matrix obtained by the feature matrix , a single feature matrix is obtained. The computer used in this simulation has a $2.9 \mathrm{GHz}$ Core $i 7$ processor and 8 GB of RAM.

Table 5: Comparison of results obtained before and after optimization

\begin{tabular}{ccccc}
\hline & $\begin{array}{c}\text { Number of } \\
\text { neurons }\end{array}$ & $\begin{array}{c}\text { Classification } \\
(\%) \text { accurate rate }\end{array}$ & $\begin{array}{c}\text { The execution time of network } \\
\text { in microcontroller }\end{array}$ & $\begin{array}{c}\text { Occupied } \\
\text { space (byte) }\end{array}$ \\
\hline $\begin{array}{c}\text { Non-optimized } \\
\text { (14 features }) \\
\begin{array}{c}\text { Optimized } \\
\text { (single feature })\end{array}\end{array}$ & 20 & $\mathbf{9 9 . 4 5}$ & 2.21 & 1340 \\
\hline
\end{tabular}

According to Table 5, the optimized results reduce the network execution time and space occupied in the microcontroller.

\section{REFERENCES}

1. Gurudath, N. and Riley, H.B. (2014),“ Drowsy Driving Detection by EEG Analysis Using Wavelet Transform and K-Means Clustering”, The 11th International Conference on Mobile Systems and Pervasive Computing, Procedia Computer Science 34 (2014) 400 - 409.

2. Kaur, R.K. and Kaur, G. (2013), "Neural Network Based Drowsiness Detection Using Electroencephalogram", International Journal of Engineering Research \& Technology (IJERT), Vol. 2 Issue 10, October - 2013. ISSN: 2278-0181.

3. Blanco, A.D. and Ramirez, R. (2019), "Evaluation of a Sound Quality Visual Feedback System for Bow Learning Technique in Violin Beginners", An EEG Study. Front. Psychol. 10:165. doi: 10.3389/fpsyg.2019.0016.

4. Asadnejad, M. sarabi, s., rajebi, s. 2019. The investigation of the different methods of clustering based on artificial intelligence to classify the malignancy of cancer lymph. The fifth international conference on innovation and research in engineering sciences. Georgia, Tiflis

5. Graupe, D. (2007), "Principles of artificial neural networks", world scientific publishing co. Pte. LTD., Vol.6, second edition.

6. Asadnejad, M. and Sarabi, S. and Rajebi, S. (2019), "Classification of Metastases or Malign Lymph by Lymphogram Results Using Bayesian, K-Nearest Neighbor and Artificial Neural Networks", 15th International Conference on Technical and Physical Problems of Electrical Engineering, Istanbul, Turkey.

7. McDonald, R. and Hall, K. and Mann, G. (2010), "Distributed Training Strategies for the Structured Perceptron”. pp. 456-464. Association for Computational Linguistics.

8. Strizi, L. and Pervan, B. and Knezovic, J. (2019), "Deep Learning Accelerator on Programmable Heterogeneous System with RISC-V Processor”, MIPRO 2019/CTS, 1126-1131.

9. Kheiri, s. and Yousefi, v. and Rajebi, S. (2019), "CLASSIFICATION WITH USING K-NEAREST NEIGHBOR, BAYESIAN AND NEURAL NETWORK METHODS IN DERMATOLOGY DISEASE”, 15th 
International Conference on Technical and Physical Problems of Electrical Engineering, Istanbul, Turkey.

10. Buhmann, M.D. (2003), "Radial Basis Functions" Theory and Implementations. Cambridge University. ISBN 0-521-63338-9.

11. Borghate, R.P. and Gosavi, S.K. (2014), "Frequency Offset Compensation in OFDM System Using Neural Network", International Journal of Advanced Research in Electrical, Electronics and Instrumentation Engineering, Vol. 3, Issue 7.

12. Lemu, H.G. and Trzepieciński, T. and Kubit, A. and Fejkie, R. (2017), "FRICTION MODELING OF AL-MG ALLOY SHEETS BASED ON MULTIPLE REGRESSION ANALYSIS AND NEURAL NETWORKS", Advances in Science and Technology Research Journal, Volume 11, Iss. 1, March 2017, pages 48-57, DOI: 10.12913/22998624/68460.

13. Wang, S. and Li, D. and Wei, Y. and Li, H. (2009), "A Feature Selection Method Based on Fisher's Discriminant Ratio for Text Sentiment Classification”, WISM, LNCS 5854, pp. 88-97.
14. Marathe, A. and Vyas, V. and Rege, P. (2017), "Inverse Fisher Discriminant Ratio based Training Set Selection for Optimal Classification Accuracy", International Journal of Applied Engineering Research, Volume 12, Number 20, pp. 9535-9541. ISSN 0973-4562.

15. Lin, T.H. and Li, H.T. and Tsai, K.C. (2003), "Implementing the Fisher's Discriminant Ratio in a k-Means Clustering Algorithm for Feature Selection and Data Set Trimming", American Chemical Society, 44, 76-87.

16. Rahmani, O., Taherkhani, A., Rahmani, M., \& Karimiyan, T. (2014). The optimal design of earthing system based on genetic algorithm. Advances in Environmental Biology, 644-657.

17. Davis, L. (1991) Handbook of Genetic Algorithms, Van Nostrand Reinhold, New York. 